05.1

\title{
Учет влияния полей остаточных деформаций в современных физико-механических технологиях обработки конструкционных материалов
}

\author{
(C) Ю.Н. Кульчин, В.Е. Рагозина, О.В. Дудко \\ Институт автоматики и процессов управления ДВО РАН, Владивосток, Россия \\ I E-mail: dudko@iacp.dvo.ru
}

Поступило в Редакцию 2 августа 2018 г.

\begin{abstract}
Для нелинейной градиентной модели больших упругопластических деформаций дано строгое определение механизмов перераспределения предварительно накопленных необратимых деформаций в результате дополнительных упругих ударных воздействий на материал. Показано, что такое перераспределение ограничивается жестким переносом и вращением тензора пластических деформаций. Получены формулы изменения начальных значений компонент тензора пластических деформаций в упругих волнах. Показано, что предварительное пластическое поле влияет на динамику дальнейшего обратимого деформирования как один из факторов создания начального квазистатического упругого поля, которое невозможно получить в чисто упругом процессе.
\end{abstract}

DOI: 10.21883/PJTF.2019.01.47152.17487

Развитие эффективных технологий интенсивной обработки деталей конструкций основано на использовании современного лазерного и электронно-лучевого оборудования [1-3] и дополняется традиционными методами термомеханического импульсного воздействия. Разработка таких технологий невозможна без создания теоретических нелинейных моделей и их верификации по накопленным экспериментальным данным. В частности, согласно общим положениям механики сплошных сред, моделирование деформационного поведения твердых тел при лазерной обработке обязательно должно одновременно учитывать и геометрическую, и физическую нелинейность связей напряжений, деформаций и температуры. Нелинейные модели более тонко отражают взаимодействие полей остаточных внутренних напряжений и деформаций с акустическими волнами, возникающими в материале наряду с локальной областью максимального поглощения энергии лазерного или ионизирующего излучения. Известно [4], что такие волны создают „эффекты дальнодействия“, которые могут приводить к существенным изменениям геометрии поверхности конструкции. Цель настоящей работы заключается в выявлении причин указанных деформационных эффектов для последующей разработки оптимальных воздействий, позволяющих получать детали с необходимыми свойствами и требуемой геометрией. Для этого определим механизмы перераспределения необратимых деформаций, а также укажем степень влияния предварительного пластического поля на последующие упругие процессы в рамках нелинейной градиентной модели больших упругопластических деформаций [5], активно развивающейся в настоящее время.

Основное предположение модели [5] - разделение левого тензора деформаций Альманзи А на упругую $(\mathbf{E})$ и пластическую $(\mathbf{P})$ части согласно представлению

$$
\begin{gathered}
2 \mathbf{A}=\mathbf{I}-\boldsymbol{\Phi}^{-1 \mathrm{~T}} \cdot \boldsymbol{\Phi}^{-1}=\mathbf{I}-\mathbf{V}^{-2}=\mathbf{I} \\
-(\mathbf{I}-\mathbf{E}) \cdot(\mathbf{I}-2 \mathbf{P}) \cdot(\mathbf{I}-\mathbf{E}), \\
\mathbf{E}=\mathbf{E}^{\mathrm{T}}, \quad \mathbf{P}=\mathbf{P}^{\mathrm{T}}, \quad \mathbf{V}=\mathbf{V}^{\mathrm{T}}, \\
\mathbf{\Phi}=\mathbf{r}_{i} \otimes \mathbf{R}^{i}=\mathbf{V} \cdot \mathbf{S}, \quad \mathbf{r}_{i}=\left.\frac{\partial \mathbf{r}}{\partial a^{i}}\right|_{t}, \quad \mathbf{R}_{i}=\left.\frac{\partial \mathbf{r}}{\partial a^{i}}\right|_{T}, \\
\mathbf{R}^{i} \cdot \mathbf{R}_{j}=\delta_{j}^{i}, \quad \mathbf{S} \cdot \mathbf{S}^{\mathrm{T}}=\mathbf{I},
\end{gathered}
$$

где $\boldsymbol{\Phi}$ - базовый тензор-градиент деформаций ( $\otimes-$ операция внешнего тензорного произведения); V - левый тензор искажений; I - метрический тензор; $\mathbf{E}$ - базовый тензор для упругих деформаций $\boldsymbol{\varepsilon}=\mathbf{E}-\mathbf{E}^{2} / 2 ; \mathbf{r}\left(a^{i}, t\right)$ - радиус-вектор точки среды в евклидовом пространстве; $a^{i}$ - координаты Лагранжа; моментам $t \geq 0$ и $T<0$ соответствуют актуальная и свободная конфигурации. Представление (1) позволяет учесть взаимодействия $\mathbf{E}$ и $\mathbf{P}$ на различных стадиях деформирования. При этом ставим основную цель указать механизм изменения $\mathbf{E}$ и $\mathbf{P}$ в среде, где в момент $t=0$ существуют предварительные статические деформации $\mathbf{E}^{\circ} \neq 0$ и $\mathbf{P}^{\circ} \neq 0$, а при $t \geq 0$ происходит дополнительное упругое деформирование. Для этого определим промежуточную конфигурацию $\mathbf{r}^{\circ}=\mathbf{r}\left(a^{i}, T_{0}\right)$ и связанные с ней тензоры-градиенты деформаций $\mathbf{F}$ и $\mathbf{\Psi}$

$$
\begin{gathered}
\mathbf{r}_{i}^{\circ}=\frac{\partial \mathbf{r}^{\circ}}{\partial a^{i}}=\left.\frac{\partial \mathbf{r}}{\partial a^{i}}\right|_{T_{0}}, \quad \mathbf{F}=\mathbf{r}_{i}^{\circ} \otimes \mathbf{R}^{i}, \quad \boldsymbol{\Psi}=\mathbf{r}_{i} \otimes \mathbf{r}^{\circ i}, \\
\mathbf{u}(\mathbf{r}, t)=\mathbf{h}(\mathbf{r}, t)+\mathbf{u}^{\circ}(\mathbf{r}-\mathbf{h}(\mathbf{r}, t)), \\
\boldsymbol{\Psi}^{-1 \mathrm{~T}}=\mathbf{I}-\nabla \otimes \mathbf{h}, \quad \mathbf{H}=\nabla \otimes \mathbf{h}=h_{, i}^{j} \mathbf{r}^{i} \otimes \mathbf{r}_{j}, \\
h_{, i}^{j}=\frac{\partial h^{j}}{\partial a^{i}}+\Gamma_{i k}^{j} h^{k},
\end{gathered}
$$




$$
\mathbf{I}-2 \mathbf{A}=\mathbf{\Psi}^{-1 \mathrm{~T}} \cdot\left(\mathbf{I}-2 \mathbf{A}^{\circ}\right) \cdot \mathbf{\Psi}^{-1} .
$$

В (2) $T_{0} \geq t=0-$ момент вовлечения точки среды вместе с ее локальной окрестностью в новый упругий процесс. Поскольку актуальная конфигурация среды выбрана в качестве основной, $\mathbf{h}(\mathbf{r}, t)$ - дополнительные перемещения, создаваемые упругим процессом при $t \geq T_{0}$, $\mathbf{u}^{\circ}(\mathbf{r}-\mathbf{h}(\mathbf{r}, t))$ - поле ранее созданных квазистатических перемещений. Из (2) для тензора Альманзи А с учетом его промежуточного значения $\mathbf{A}^{\circ}$ получим

$$
\begin{aligned}
& \mathbf{A}=\boldsymbol{\varepsilon}+\mathbf{P}-\mathbf{E} \cdot \mathbf{P}-\mathbf{P} \cdot \mathbf{E}+\mathbf{E} \cdot \mathbf{P} \cdot \mathbf{E}=\boldsymbol{\varepsilon}^{\circ} \\
& +\frac{1}{2}\left(\mathbf{H}+\mathbf{H}^{\mathrm{T}}-\mathbf{H} \cdot \mathbf{H}^{\mathrm{T}}\right)-\mathbf{H} \cdot \varepsilon^{\circ}-\varepsilon^{\circ} \cdot \mathbf{H}^{\mathrm{T}}+\mathbf{H} \cdot \varepsilon^{\circ} \cdot \mathbf{H}^{\mathrm{T}} \\
& +\mathbf{P}^{\circ}-\mathbf{P}^{\circ} \cdot\left(\mathbf{E}^{\circ}+\mathbf{H}^{\mathrm{T}}-\mathbf{E}^{\circ} \cdot \mathbf{H}^{\mathrm{T}}\right)-\left(\mathbf{E}^{\circ}+\mathbf{H}-\mathbf{H} \cdot \mathbf{E}^{\circ}\right) \cdot \mathbf{P}^{\circ} \\
& +\mathbf{D}^{\circ}+\mathbf{H} \cdot \mathbf{P}^{\circ} \cdot \mathbf{E}^{\circ}-\mathbf{H} \cdot \mathbf{D}^{\circ}+\mathbf{E}^{\circ} \cdot \mathbf{P}^{\circ} \cdot \mathbf{H}^{\mathrm{T}}-\mathbf{D}^{\circ} \cdot \mathbf{H}^{\mathrm{T}} \\
& +\mathbf{H} \cdot \mathbf{P}^{\circ} \cdot \mathbf{H}^{\mathrm{T}}-\mathbf{H} \cdot \mathbf{E}^{\circ} \cdot \mathbf{P}^{\circ} \cdot \mathbf{H}^{\mathrm{T}}-\mathbf{H} \cdot \mathbf{P}^{\circ} \cdot \mathbf{E}^{\circ} \cdot \mathbf{H}^{\mathrm{T}} \\
& +\mathbf{H} \cdot \mathbf{D}^{\circ} \cdot \mathbf{H}^{\mathrm{T}}, \\
& \text { где } \quad \quad \mathbf{D}^{\circ}=\mathbf{E}^{\circ} \cdot \mathbf{P}^{\circ} \cdot \mathbf{E}^{\circ}, \quad \mathbf{E}^{\circ}=\mathbf{E}^{\circ}(\mathbf{r}-\mathbf{h}(\mathbf{r}, t)), \\
& \mathbf{P}^{\circ}=\mathbf{P}^{\circ}(\mathbf{r}-\mathbf{h}(\mathbf{r}, t)) .
\end{aligned}
$$

Если изменение тензора $\mathbf{P}$ в упругом процессе связано только с его жестким поворотом, то

$$
\mathbf{P}=\mathbf{C} \cdot \mathbf{P}^{\circ} \cdot \mathbf{C}^{\mathrm{T}}, \quad \mathbf{C} \cdot \mathbf{C}^{\mathrm{T}}=\mathbf{C}^{\mathrm{T}} \cdot \mathbf{C}=\mathbf{I}, \quad \mathbf{C}=\mathbf{I}+\mathbf{W} .
$$

Подставляя (4) в (3) и утверждая, что упругая деформация $\varepsilon$ зависит от своего начального значения $\varepsilon^{\circ}$ и поля $\mathbf{H}$ и не зависит от уровня предварительных значений $\mathbf{P}^{\circ}$, для тензоров $\boldsymbol{\varepsilon}, \mathbf{W}$ и $\mathbf{C}$ нетрудно получить

$$
\begin{aligned}
& \begin{array}{r}
\boldsymbol{\varepsilon}= \\
=
\end{array} \mathbf{E}-\frac{\mathbf{E}^{2}}{2}=\boldsymbol{\varepsilon}^{\circ}+\frac{1}{2}\left(\mathbf{H} \cdot\left(\mathbf{I}-2 \boldsymbol{\varepsilon}^{\circ}\right)\right. \\
&\left.+\left(\mathbf{I}-2 \boldsymbol{\varepsilon}^{\circ}\right) \cdot \mathbf{H}^{\mathrm{T}}-\mathbf{H} \cdot\left(\mathbf{I}-2 \boldsymbol{\varepsilon}^{\circ}\right) \cdot \mathbf{H}^{\mathrm{T}}\right), \\
& \mathbf{W}=(\mathbf{I}-\mathbf{E})^{-1} \cdot\left(\mathbf{E}-\mathbf{E}^{\circ}+\mathbf{H} \cdot \mathbf{E}^{\circ}-\mathbf{H}\right), \\
& \mathbf{C}=\mathbf{I}+\mathbf{W}=(\mathbf{I}-\mathbf{E})^{-1} \cdot(\mathbf{I}-\mathbf{H}) \cdot\left(\mathbf{I}-\mathbf{E}^{\circ}\right) .
\end{aligned}
$$

Тензор $\mathbf{C}$ в (5) является ортогональным $\left(\mathbf{C} \cdot \mathbf{C}^{\mathrm{T}}=\right.$ $\left.=\mathbf{C}^{\mathrm{T}} \cdot \mathbf{C}=\mathbf{I}\right)$, поэтому задает жесткое вращение $\mathbf{P}^{\circ}$. $\mathbf{C o}^{-}$ отношения (4), (5) обращают (3) в тождество. Нетрудно проверить, что, предполагая только жесткое вращение $\mathbf{P}^{\circ}$, из связи (2) между $\mathbf{A}, \boldsymbol{\Psi}^{-1}$ и $\mathbf{A}^{\circ}$ получим представление (5) для тензора С. Далее условие ортогональности $\mathbf{C}$ определяет связь $\varepsilon \mathbf{c ~} \mathbf{H}, \varepsilon^{\circ}$ через первую формулу (5). Таким образом, исходное утверждение для $\varepsilon$ доказано. Для исключения возможности существования другого решения задачи (иной связи $\varepsilon$ с $\varepsilon^{\circ}, \mathbf{P}^{\circ}, \mathbf{H}$ ) проведем доказательство в обратную сторону. Из (2) следует

$$
\begin{aligned}
\mathbf{I}-2 \mathbf{A}=(\mathbf{I}-\mathbf{E}) \cdot(\mathbf{I}-2 \mathbf{P}) \cdot(\mathbf{I}-\mathbf{E}) \\
=\mathbf{\Psi}^{-1 \mathrm{~T}} \cdot\left(\mathbf{I}-\mathbf{E}^{\circ}\right) \cdot\left(\mathbf{I}-2 \mathbf{P}^{\circ}\right) \cdot\left(\mathbf{I}-\mathbf{E}^{\circ}\right) \cdot \mathbf{\Psi}^{-1} \\
=(\mathbf{I}-\mathbf{H}) \cdot\left(\mathbf{I}-\mathbf{E}^{\circ}\right) \cdot\left(\mathbf{I}-2 \mathbf{P}^{\circ}\right) \cdot\left(\mathbf{I}-\mathbf{E}^{\circ}\right) \cdot\left(\mathbf{I}-\mathbf{H}^{\mathrm{T}}\right) \\
=(\mathbf{I}-\mathbf{E}) \cdot \mathbf{C} \cdot\left(\mathbf{I}-2 \mathbf{P}^{\circ}\right) \cdot \mathbf{C}^{\mathrm{T}} \cdot(\mathbf{I}-\mathbf{E}), \\
\mathbf{C} \cdot\left(\mathbf{I}-2 \mathbf{P}^{\circ}\right) \cdot \mathbf{C}^{\mathrm{T}}=(\mathbf{I}-\mathbf{E})^{-1} \cdot(\mathbf{I}-\mathbf{H}) \cdot\left(\mathbf{I}-\mathbf{E}^{\circ}\right) \cdot\left(\mathbf{I}-2 \mathbf{P}^{\circ}\right) \\
\quad \times\left(\mathbf{I}-\mathbf{E}^{\circ}\right) \cdot\left(\mathbf{I}-\mathbf{H}^{\mathrm{T}}\right) \cdot(\mathbf{I}-\mathbf{E})^{-1}, \quad(6)
\end{aligned}
$$

что позволяет определить тензор $\mathbf{C}$ через $\mathbf{E}^{\circ}, \mathbf{H}, \mathbf{E}$. Условие ортогональности $\mathbf{C}$ приводит к исходному предположению (5) для тензора $\varepsilon$ и тем самым завершает наше доказательство.

Далее рассмотрим особенности движения упругих ударных волн в среде с предварительными пластическими деформациями. Для этого отметим, что в модели [5] тензор напряжений $\mathbf{T}$ Эйлера-Коши для изотропной среды связан с деформациями $\mathbf{E}, \mathbf{P}$ через квазипотенциальное соотношение

$$
\mathbf{T}=\rho \rho_{0}^{-1} U_{\mathcal{E}}(\mathbf{I}-2 \boldsymbol{\varepsilon})=\rho \rho_{0}^{-1} U_{\mathbf{E}}(\mathbf{I}-\mathbf{E}),
$$

где упругий потенциал $U-$ функция инвариантов тензора упругих деформаций $\varepsilon ; U_{\mathcal{\varepsilon}}$ и $U_{\mathbf{E}}-$ ее тензорные производные; $\rho \rho_{0}^{-1}$ - изменение плотности среды, которое может зависеть от $\mathbf{E}$ и $\mathbf{P}$ для пластически сжимаемого материала и только от $\mathbf{E}$ для пластически несжимаемой среды. Если предварительное состояние среды квазистатическое, то поле вектора скорости $\mathbf{v}$ зависит только от динамической компоненты $\mathbf{h}(\mathbf{r}, t)$. Из (7) следует, что на переднем фронте упругих ударных волн динамические условия совместности [6] не зависят от пластических деформаций $\mathbf{P}$. Поэтому возможные скорости и типы ударных волн (как и методы вычисления динамического поля $\mathbf{h}(\mathbf{r}, t))$ можно искать в работах, посвященных динамике нелинейно-упругих сред (например, [6,7]). Перераспределение пластических деформаций $\mathbf{P}^{\circ}$ и их поворот могут быть вычислены по найденному полю $\mathbf{h}(\mathbf{r}, t)$, что является исключительно удобным при реализации численных схем. Для вычисления поворотов тензора $\mathbf{P}^{\circ}$ дополнительным важным свойством является возможность появления скачков компонент $\mathbf{P}$ на передних фронтах упругих ударных волн:

$$
\begin{gathered}
{[\mathbf{P}]=[\mathbf{C}] \cdot \mathbf{P}^{\circ} \cdot\left(\mathbf{C}^{\mathrm{T}}\right)^{+}+\mathbf{C}^{+} \cdot \mathbf{P}^{\circ} \cdot\left[\mathbf{C}^{\mathrm{T}}\right]-[\mathbf{C}] \cdot \mathbf{P}^{\circ} \cdot\left[\mathbf{C}^{\mathrm{T}}\right],} \\
{[\mathbf{C}]=\mathbf{C}^{+}-\mathbf{C}^{-},}
\end{gathered}
$$

причем скачкообразный поворот $[\mathbf{C}]$ вычисляется через $[\mathbf{E}],[\mathbf{H}]$, согласно (5).

Относительно влияния пластических деформаций на упругие процессы отметим следующее. С одной стороны, пластические деформации не входят в краевые условия динамической задачи и их наличие в скалярном 


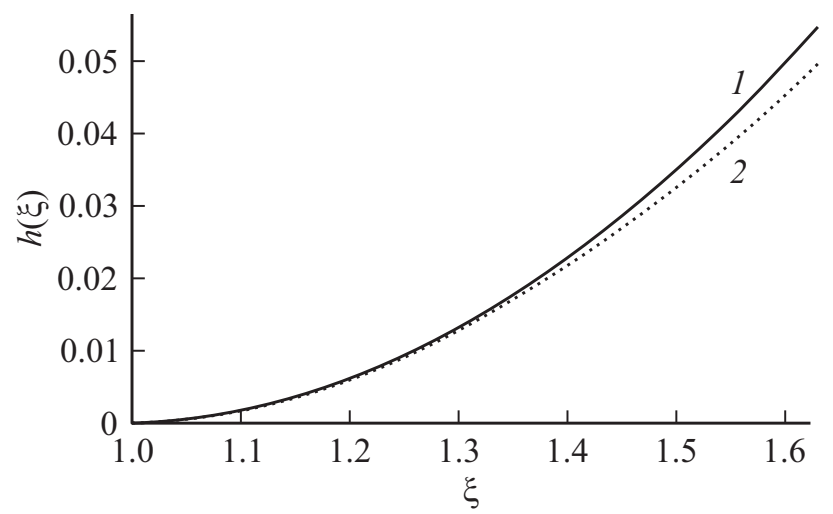

Рис. 1. Мгновенное распределение поля $h(\xi)$ в прифронтовой окрестности упругой ударной волны $(\xi=1)$ в момент $T_{1}=10^{-6} \mathrm{~s} .1-$ численное решение, 2 - аналитическое приближение.

множителе $\rho \rho_{0}^{-1}$ формулы (7) зависит от модели пластичности. С другой стороны, пластические деформации косвенно влияют на интегрирование уравнений движения относительно вектора $\mathbf{h}$ и упругих деформаций $\mathbf{E}$ и $\varepsilon$ из-за наличия в этих уравнениях $\varepsilon^{\circ}$. В общем случае компоненты $\varepsilon^{\circ}$ не удовлетворяют условиям совместности деформаций и определяемое с их участием поле $\mathbf{h}(\mathbf{r}, t)$ не имеет аналога в решениях чисто упругих задач. Если граница деформируемого материала заранее неизвестна, то ненулевой тензор $\mathbf{P}^{\circ}$ окажет влияние и на ее вычисление.

Проиллюстрируем применение изложенных общих соотношений на максимально простом примере движения сферической продольной упругой ударной волны сжатия от границы сферического дефекта. Полагаем, что в области $r \geq r_{0}+l(t) \quad\left(r_{0} \neq 0, l(t) \geq 0, l(0)=0\right)$ до момента $t=0$ при $e_{r r}^{\circ}=e_{\phi \phi}^{\circ}=e_{\theta \theta}^{\circ}=0$ имеются ненулевые $p_{r r}^{\circ}$ и $p_{\phi \phi}^{\circ}=p_{\theta \theta}^{\circ}$. Дополнительно считаем, что $\left.\rho \rho_{0}^{-1}\right|_{t \leq 0}=K_{0}=$ const $<1$ (предварительное статическое растяжение) и ударная волна движется с постоянной скоростью $G=$ const. Сохраняя в (7) только линейные слагаемые по компонентам $\mathbf{E}$ и переходя в безразмерное пространство $\xi=r^{-1}\left(r_{0}+G t\right)$, получаем уравнение движения в форме нелинейного обыкновенного дифференциального уравнения относительно $h(\xi)=r^{-1} h_{r}(r, t)$

$$
\begin{gathered}
h^{\prime \prime}\left\{\xi^{2}-\left(1+h^{\prime}(1)\right)(1-h)^{4}\left(1-h+\xi h^{\prime}\right)^{-2}\right\}=2 \xi h^{\prime}, \\
h(1)=0, \quad h^{\prime}(1)=\tau>0, \quad \xi \geq 1, \quad \tau \ll 1 .
\end{gathered}
$$

Решение уравнения (8) в прифронтовой области ударной волны $(\xi=1$, рис. 1$)$ может быть равноправным образом получено как численно, так и аналитически методом малого параметра в растянутых координатах $x=\tau^{-1}(\xi-1), x \geq 0, g=\tau^{-2} h, g^{\prime}(0)=1$. На рис. 2 представлено соответствующее такому решению перераспределение предварительных пластических деформа- ций за упругой ударной волной в момент $T_{1}>0$

$$
\begin{gathered}
p_{r r}\left(r, T_{1}\right)=\left(1-K_{0}^{2} w^{-4 / 3}\right) / 2, p_{\phi \phi}\left(r, T_{1}\right)=\left(1-w^{2 / 3}\right) / 2, \\
w\left(r, T_{1}\right)=K_{0}+r_{0}^{3}\left(r-h_{r}\left(r, T_{1}\right)\right)^{-3}\left(\left(1-u^{\circ} r_{0}^{-1}\right)^{3}-K_{0}\right), \\
u^{\circ}=\left.u_{r}^{\circ}\right|_{r=r_{0}} .
\end{gathered}
$$

Из рис. 2 следует, что для рассмотренного максимально простого типа краевой задачи (когда $\mathbf{P}$ не испытывает поворота и перераспределяется только за счет нового упругого перемещения среды) наиболее существенные изменения начального поля $p_{r r}^{\circ}, p_{\phi \phi}^{\circ}=p_{\theta \theta}^{\circ}$ происходят в области больших градиентов предварительных пластических деформаций в окрестности $r=r_{0}$. При этом для каждой точки пространства, вовлеченной в упругий процесс, уровень $p_{r r}$ возрастает относительно $p_{r r}^{\circ}$, а уровень $p_{\phi \phi}$ заметно снижается относительно исходного $p_{\phi \phi}^{\circ}$. Данный эффект невозможно учесть в рамках линейной упругопластичности. Одновременно заметим, что если бы упругопластическая начальная область была ограничена с двух сторон $\left(r_{0} \leq r \leq r^{*}\right)$, то ее новое положение было бы $r_{0}+l(t) \leq r \leq r^{*}+u^{*}(t)$.

Таким образом, по сравнению с данными работы [8], где изменения пластических деформаций в рамках модели [5] вычислялись для квазистатических задач с привлечением дополнительных предположений (несжимаемость среды, анализ малости и т.д.), полученное
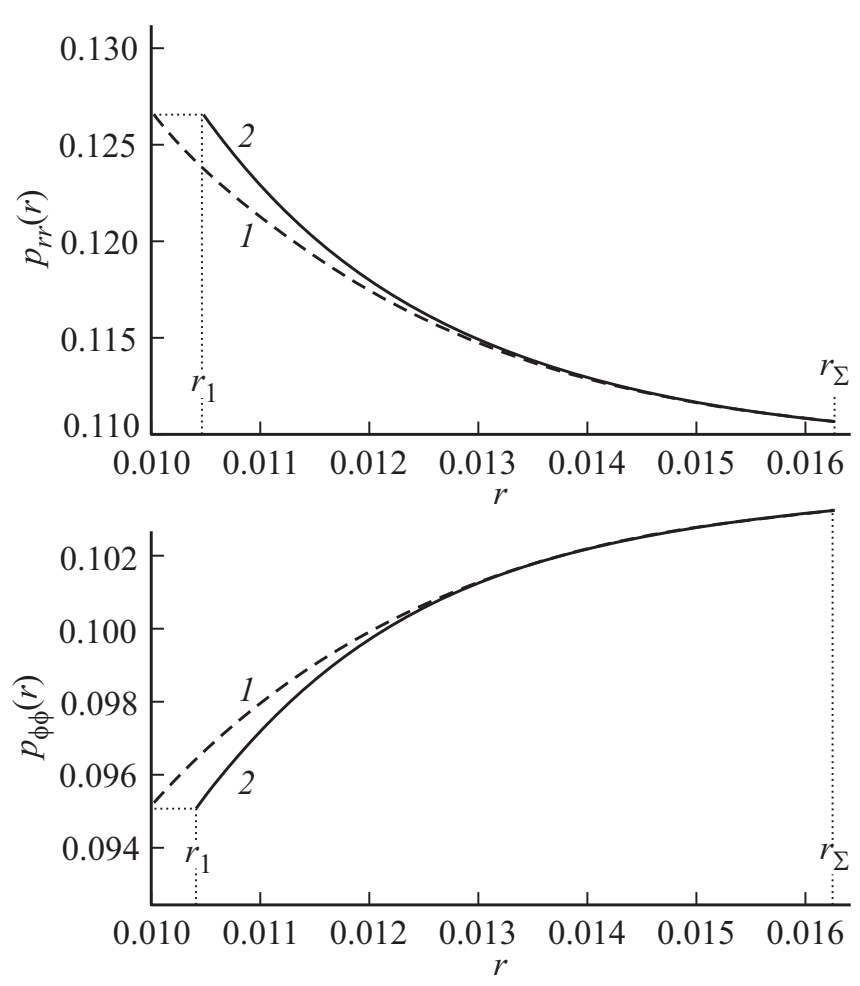

Рис. 2. Перераспределение предварительного поля пластических деформаций: 1 - начальные $p_{r r}^{\circ}, p_{\phi \phi}^{\circ}=p_{\theta \theta}^{\circ}$ в момент $t=0,2-$ актуальные $p_{r r}, p_{\phi \phi}=p_{\theta \theta}$ в момент $T_{1}=10^{-6} \mathrm{~s}$. $r_{1}, r_{\Sigma}-$ мгновенные координаты подвижной границы сферического дефекта и фронта ударной волны соответственно. 
представление (3)-(6) обладает существенными преимуществами: 1) это строгий универсальный алгоритм описания произвольной кинематики материала при любых методах его обработки; 2) здесь нет необходимости (как и следовало ожидать для упругих процессов) обращаться к скоростным характеристикам среды (скорости деформаций), так как решение проводится на уровне тензоров деформаций; 3) из (3)-(6) можно получить важные качественные выводы для упругой динамики упругопластической среды.

Работа выполнена в рамках госбюджетной темы № AАAА-A17-117040450015-0 и при частичной финансовой поддержке Министерства образования и науки РФ (постановление П218, договор № 02.G25.31.0116 от 14.08.2014 г. между ОАО «Центр судоремонта „Дальзавод“» и Министерством образования и науки РФ).

\section{Список литературы}

[1] Шибков А.А., Золотов А.Е., Гасанов М.Ф., Желтов М.А., Проскуряков К.А. // Письма в ЖТФ. 2015. Т. 41. В. 24. C. 70-76.

[2] Песчанская Н.Н., Смолянский А.С., Шведов А.С. // ФТТ. 2009. T. 51. B. 6. C. $1218-1222$.

[3] Заярный Д.А., Ионин А.А., Кудряшов С.И., Макаров С.В., Кучмижск А.А., Витрик О.Б., Кульчин Ю.Н. // Письма в ЖЭТФ. 2016. Т. 103. В. 12. С. 846-850.

[4] Овчинников В.В., Гущина Н.В., Романов И.Ю., Кайгородова Л.И., Григорьев А.Н., Павленко А.В., Плохой В.В. // Изв. вузов. Физика. 2016. Т. 59. № 10. С. 3-8.

[5] Быковцев Г.И., Шитиков А.В. // ДАН СССР. 1990. Т. 311. № 1. C. 59-62.

[6] Бленд Д.Р. Нелинейная динамическая теория упругости. М.: Мир, 1972. 183 с.

[7] Куликовский А.Г., Свешникова Е.И. Нелинейные волны в упругих средах. М.: Московский лицей, 1998. 412 с.

[8] Буренин А.А., Ковтанюк Л.В. Большие необратимые деформации и упругое последействие. Владивосток: Дальнаука, 2013. 312 с. 